UDK 635.054:718(497.11 Obrenovac)

Original scientific paper

\title{
THE STATE AND FUNCTIONALITY OF DENDROFLORA IN THE CEMETERIES IN OBRENOVAC
}

\author{
Suzana MITROVIĆl, Nevena ČULE ${ }^{1}$, Dušan JOKANOVIĆ ${ }^{2}$, Milijana CVEJIĆ ${ }^{l}$, \\ Milorad VESELINOVIĆl
}

\begin{abstract}
Urban cemeteries represent important human-made special-purpose green areas. On the territory of the city area of the Municipality of Obrenovac, there are two urban cemeteries: the New Cemetery (Serbian: Novo Groblje) and the Old Cemetery (Serbian: Staro Groblje). The New Cemetery with an area of 5.4 ha is at $2.84 \mathrm{~km}$ west of the center and Staro Groblje with an area of 4.5 ha lies at $2 \mathrm{~km}$ from the city center.

In the framework of the development of the Cadastre of Public Green Areas of the Municipality of Obrenovac, 29 woody species with a total of 271 trees were recorded in the New Cemetery. The Old Cemetery had 32 woody species recorded with a total of 348 trees. The paper studies the vitality, the ornamental value, as well as the functionality and adaptability of the species to the existing environmental conditions.
\end{abstract}

ornamental value.

Keywords: dendroflora, Obrenovac, urban cemeteries, adaptability, vitality,

\footnotetext{
${ }^{1}$ Dr Suzana Mitrović, Dr Nevena Čule, Mr Milijana Cvejić, Dr Milorad Veselinović Institute of forestry, 3 Kneza Viseslava, Belgrade, Serbia

${ }^{2}$ Dr Dušan Jokanović, Faculty of Forestry Belgrade, University of Belgrade, 1 Kneza Višeslava, Serbia Corresponding author: Suzana Mitrović, 3 Kneza Višeslava, 11030 Belgrade, Serbia, tel.: +381 62 8838024, email: mitrovicsuzana@yahoo.com
}

Acknowledgement: The research was partly financed by the Ministry of Education and Science, Republic of Serbia, within the Project 'The study of climate change on the environment: monitoring impacts, adaption and mitigation' III 43007 (2010-2018). 


\title{
STANJE I FUNKCIONALNOST DENDROFLORE NA GROBLJIMA U OBRENOVCU
}

\begin{abstract}
Abstrakt: Gradska groblja predstavljaju značajne antropogene zelene površine specijalne namene. Na teritoriji gradskog područja opštine Obrenovac nalaze se dva gradska groblja: Novo i Staro groblje. $\mathrm{Na} 2,84 \mathrm{~km}$ zapadno od centra nalazi se Novo groblje, površine 5,4 ha, na 2 km Staro groblje površine 4,5 ha.

U okviru izrade Katastra javnih zelenih površina gradske opštine Obrenovac, na Novom groblju evidentirano je 29 drvenastih vrsta sa ukupno 271 stablom. Na Starom groblju evidetirane su 32 drvenaste vrste sa ukupno 348 stabala.

U radu je izvršena analiza vitalnosti, dekorativnosti, funkcionalnosti i adaptivnosti korišćenih vrsta na postojeće uslove sredine.
\end{abstract}

Ključne reči: dendroflora, Obrenovac, gradska groblja, adaptivnost, vitalnost, dekorativnost.

\section{INTRODUCTION}

Cemeteries are important elements of urban areas. Apart from cherishing the memory of the deceased and buried, urban cemeteries also represent important special-purpose green areas (Anastasijević, 2002; Vujković, 2003) with multiple benefits in terms of environmental protection. These areas, connected with other green areas of different categories, constitute the green structure of the city whose functions contribute to the healthier environment of the polluted urban area. The development of the Cadastre of Public Green Areas of the urban municipality of Obrenovac (Veselinović et al., 2010) included the inventory of the urban cemetery dendroflora and the study of its state and the functionality.

There are two urban cemeteries on the territory of the urban area of the Municipality of Obrenovac - the New Cemetery and the Old Cemetery.

\section{MATERIAL AND METHODS}

Within the field surveys conducted in the area of the urban cemeteries, trees were identified in the field and recorded as point objects.

GPS locations were determined using a Trimble ${ }^{\circledR}$ GeoExplorer ${ }^{\circledR}$ series GPS device with the Microsoft ${ }^{\circledR}$ Windows Mobile ${ }^{\mathrm{TM}} 2003$ software platform which communicates with the TruPulse $360 \mathrm{~B}$ laser distance meter produced by Laser Technology Inc. These two devices are paired using a Bluetooth connection. The Trimble licensed TerraSync ${ }^{\mathrm{TM}}$ software was used for the purpose of data collection. The type of greenery and the species of trees were determined in the field. If there were any doubts, dendrological material was collected (a leaf and a twig), and the species was subsequently determined using the identification key and literature data (Hessayonu, 2001; Hillier, 1982; Idžojtić 2009; Jovanović, 1985; Ocokoljić and Ninić-Todorović, 2003; Vidaković, 1982; Vukićević, 1996; Ward, 2001; Šijak, 2000). 
The heights of trees, as well as the heights of trunks and the spread of crowns were measured using the TruPulse 360 B laser distance meter (Veselinović et al., 2014).

The diameter of all trees was measured at 'breast` height $(1.30 \mathrm{~m})$, using a caliper gauge. The diameter was determined as the arithmetic mean of two crossmeasured tree diameters.

The presence and the type of damage were determined on the basis of the symptoms observed (Ćirković-Mitrović et al., 2016). The damage was classified as mechanical, physiological, entomological or phytopathological based on which the vitality and the ornamental value were assessed.

The vitality of each individual tree was assessed according to the following evaluation scale by the method (literature):

1 - a seriously diseased, damaged or dead tree

2 - a severely diseased or damaged tree or a tree affected by decaying processes but still alive

3 - a vital tree that needs regular tending measures to survive

4 - a vital tree that needs occasional tending to survive

5 - a completely healthy tree, or a tree of exceptional vitality

Each individual tree was further assigned an ornamental value rating. In accordance with the standard characteristics specific for each individual species, the following method was used for the evaluation (literature):

$1-$ a tree without ornamental features

$2-$ a tree with poor ornamental features

3 - a tree with a standard habitus but with some defects

$4-\mathrm{a}$ tree with very good ornamental features, a regular habitus and minor defects

5 - a tree with excellent ornamental features, an extremely well-developed habitus and without any visible defects in the appearance

In exceptional cases, trees without the distinguishable characteristics of a species had their ornamental value rated high because they are interesting and draw attention with a curved trunk, twisted branches and or some other characteristics that give an added value to the landscape.

The data collected in the field were transferred from the Trimble ${ }^{\circledR}$ GeoExplorer ${ }^{\circledR}$ series GPS device to the computer. The GPS Pathfinder ${ }^{\circledR}$ Office ver. 4.20 was used for this purpose. The data that were hand-written in purposeful tables were entered into the computer using Microsoft Office Excel 2007.

\section{RESULTS AND DISCUSSION}

The municipality of Obrenovac is located in the central part of the lower Kolubara River Basin and stretches between $44^{\circ} 30^{\prime}$ and $44^{\circ} 43^{\prime} \mathrm{N}$ and $19^{\circ} 58^{\prime}$ and $20^{\circ} 20^{\prime} \mathrm{E}$. The largest part of the land is extremely flat.

The largest part of the Municipality of Obrenovac is located in the macroalluvial fan of the Kolubara River. Alluvial fans are deposits of sediment that belong to a group of accumulative fluvial forms. Macro-alluvial fans were mainly formed during the Quaternary in a particularly humid climate. Obrenovačka Posavina makes the macro-alluvial fan of the Kolubara River pushing the Sava 
River to the north about ten kilometers away from its initial flow below the Posavski Section. The macro-alluvial fan of the Kolubara with Tamnava covers a surface area of $288 \mathrm{~km}^{2}$ (Dragičević and Karić, 2007).

According to the research of the Institute of Soil Science (2009), hydromorphic soils are the most frequent on the territory of the urban municipality of Obrenovac. The area is intersected by the flows of the Sava, Kolubara, Tamnava and Peštan Rivers with the alluvial soil, humofluvisol and humogley being the dominant soil types. The land on which the cemetery was built originally had the humofluvisol, but it has been altered by human activity.

The basic climatic characteristics of the Municipality of Obrenovac are determined by its geographical position, terrain and wide openness to the Pannonian Plain. Obrenovac is located almost in the middle of the north temperate zone, with a climate milder than the typical Pannonian, continental climate. It is characterized by humid continental climate with warm summers and cold winters. The average annual temperature in this area is around $11^{\circ} \mathrm{C}$. It is around $22^{\circ} \mathrm{C}$ in summer and about $-1{ }^{\circ} \mathrm{C}$ in winter, with the maximum range between $-28^{\circ} \mathrm{C}$ and $40^{\circ} \mathrm{C}$. Because of its openness to the north, i.e., the Pannonian Plain, the effects of the continental precipitation regime on the area of Obrenovac are evident. Cyclones cause maximum precipitation in late spring and early summer, with the secondary maximum of rainfall in late autumn. The annual amount of precipitation is about 640 liters of water per square meter. It is around 440 in dry years and 940 liters per $\mathrm{m}^{2}$ in rainy years. During the year, the precipitation is highest in spring and late summer or early autumn. The study area has the surplus of water in the period from January to March, and from May, the evapotranspiration (ETP, ETR) exceeds the inflow of water from precipitation. The dry period, i.e. the water deficit (D = ETPETR) in the soil occurs in the period from June to September. In October, the water reserves in the soil are gradually being restored. Then, depending on the soil conditions and the precipitation regime, there is a surplus of water (S) which flows into rivers or groundwater either as surface runoff or through the soil (Veselinović et al., 2010).

The New Cemetery is located in the western part of Obrenovac (Figure 1), $2.84 \mathrm{~km}$ away from the city center. It is at Rvaćanska bb, in Rvati settlement. It covers an area of 5.4 ha. 


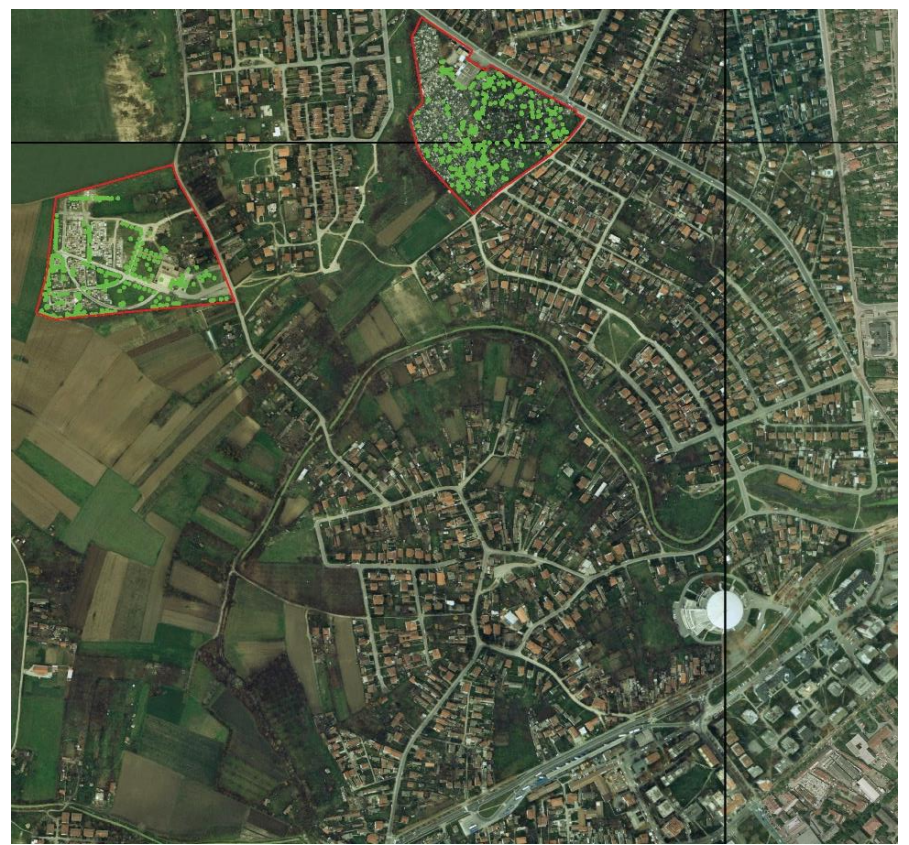

Figure 1. The location of the New Cemetery and the Old Cemetery

The Old Cemetery, measuring 4.5 hectares, is located in the western part of Obrenovac (Figure 1), $2 \mathrm{~km}$ from the city center, at 97 Nemanjina Street.

Table 1. The participation of broadleaves and conifers in the New Cemetery and

\begin{tabular}{|l|c|c|c|c|}
\multicolumn{2}{c}{ the Old Cemetery } \\
\cline { 2 - 5 } Vegetation type & \multicolumn{2}{|c|}{ The New Cemetery } & \multicolumn{2}{c|}{ The Old Cemetery } \\
\cline { 2 - 5 } & ind. & $\%$ & ind. & $\%$ \\
\hline Broadleaves & 174 & 64 & 53 & 15 \\
\hline Conifers & 97 & 36 & 295 & 85 \\
\hline Total & 271 & 100 & 348 & 100 \\
\hline
\end{tabular}

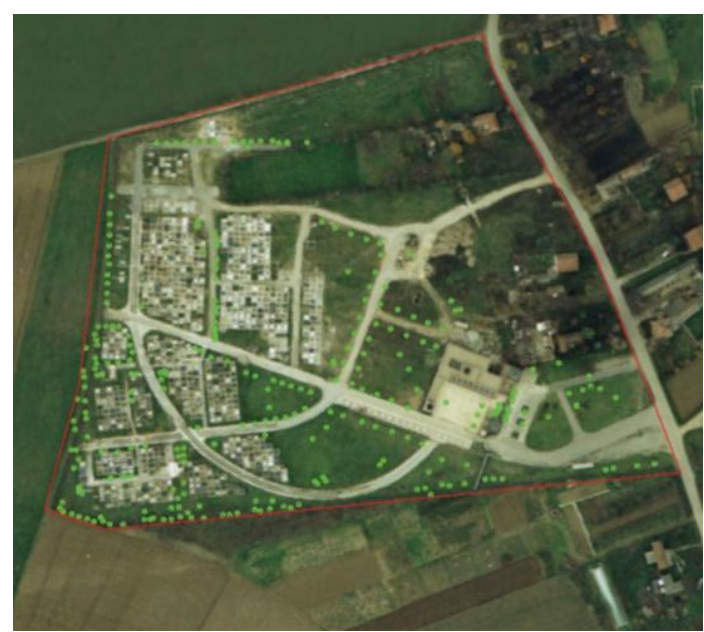

Figure 1. The position of the New Cemetary 
In the New Cemetery, 271 trees were analyzed (Figure 2), 174 of which were broadleaved and 97 coniferous species. There were 29 different tree species in total.

The most common wood species were Tilia tomentosa Mnch. with 40 individuals, Betula verrucosa Ehrh. with 37 seedlings and Chamaecyparis lawsoniana (Murr.) Parl. with 28 specimens. The ratio of the broadleaves to conifers was 64:36.

Table 2. Average values of the recorded tree parameters in the New Cemetery

\begin{tabular}{|c|c|c|c|c|c|c|c|c|}
\hline $\begin{array}{l}\text { S. } \\
n^{\circ}\end{array}$ & Species & 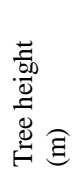 & 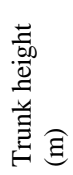 & 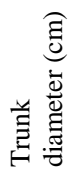 & 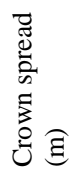 & 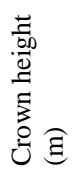 & 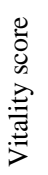 & 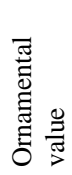 \\
\hline 1 & Abies alba Mill. & 1.5 & 0.0 & 3.0 & 1.0 & 1.5 & 4.0 & 4.0 \\
\hline 2 & Acer campestre L. & 6.5 & 1.4 & 17.5 & 3.6 & 5.1 & 4.0 & 4.0 \\
\hline 3 & Acer pseudoplatanus $\mathrm{L}$. & 3.4 & 1.2 & 10.3 & 2.2 & 2.3 & 4.0 & 3.7 \\
\hline 4 & Aesculus hippocastanum L. & 4.7 & 1.3 & 13.7 & 3.2 & 3.3 & 3.0 & 3.0 \\
\hline 5 & Betula verrucosa Ehrh. & 3.0 & 0.5 & 6.7 & 2.0 & 2.4 & 4.0 & 3.8 \\
\hline 6 & Catalpa bignonioides Walt. & 2.0 & 1.5 & 4.0 & 1.0 & 0.5 & 3.7 & 3.9 \\
\hline 7 & Cedrus atlantica Man. & 2.3 & 0.0 & 7.3 & 1.4 & 2.3 & 4.3 & 3.7 \\
\hline 8 & Cercis siliquastrum L. & 1.5 & 0.0 & 3.0 & 1.5 & 1.5 & 3.0 & 2.0 \\
\hline 9 & Chamaecyparis lawsoniana (Murr.) Parl. & 2.1 & 0.0 & 5.4 & 1.1 & 2.1 & 3.7 & 3.6 \\
\hline 10 & Cupressus sempervirens L. & 5.0 & 0.0 & 10.0 & 1.0 & 5.0 & 4.0 & 4.0 \\
\hline 11 & Fraxinus angustifolia Vahl. & 4.5 & 1.6 & 10.3 & 2.8 & 2.9 & 4.0 & 4.0 \\
\hline 12 & Juglans regia $\mathrm{L}$. & 7.0 & 1.5 & 41.0 & 7.0 & 5.5 & 3.0 & 3.0 \\
\hline 13 & Juniperus scopulorum 'Skyrocket' Sarg. & 2.7 & 0.1 & 5.4 & 0.8 & 2.6 & 3.4 & 3.9 \\
\hline 14 & Koelreuteria paniculata Laxm. & 2.0 & 0.5 & 4.0 & 1.5 & 1.5 & 4.0 & 3.0 \\
\hline 15 & Libocedrus decurrens Torr. & 2.6 & 0.0 & 8.1 & 1.2 & 2.6 & 3.4 & 4.3 \\
\hline 16 & Liriodendron tulipifera $\mathrm{L}$. & 2.3 & 1.4 & 2.9 & 0.9 & 0.9 & 3.9 & 4.0 \\
\hline 17 & Picea abies (L.) Karst. & 3.8 & 0.3 & 12.5 & 2.3 & 3.5 & 3.5 & 3.5 \\
\hline 18 & Picea omorika (Pančić) Purkyne. & 2.0 & 0.0 & 7.0 & 1.0 & 2.0 & 4.0 & 4.0 \\
\hline 19 & Picea pungens Engelm. & 2.5 & 0.0 & 5.0 & 1.2 & 2.5 & 4.0 & 3.0 \\
\hline 20 & Pinus nigra Arn. & 3.6 & 0.7 & 16.6 & 2.6 & 3.0 & 4.0 & 4.0 \\
\hline 21 & Platanus acerifolia (Ait.) Willd. & 5.0 & 1.0 & 1.5 & 4.0 & 4.0 & 5.0 & 5.0 \\
\hline 22 & Platanus occidentalis L. & 8.3 & 1.7 & 23.3 & 4.3 & 6.7 & 3.0 & 3.0 \\
\hline 23 & Prunus avium L. & 1.9 & 0.7 & 4.0 & 1.4 & 1.2 & 4.8 & 4.7 \\
\hline 24 & Prunus pissardii Ehrh. & 2.1 & 0.2 & 4.5 & 1.4 & 1.9 & 4.2 & 4.2 \\
\hline 25 & Quercus rubra Michx f. & 3.4 & 1.4 & 7.5 & 2.3 & 2.0 & 4.8 & 4.8 \\
\hline 26 & Robinia pseudoacacia L. & 3.4 & 2.0 & 3.0 & 1.0 & 1.4 & 3.8 & 3.8 \\
\hline 27 & Thuja orientalis $\mathrm{L}$. & 1.8 & 0.0 & 4.3 & 0.7 & 1.8 & 4.1 & 4.1 \\
\hline 28 & Tilia cordata Mill. & 3.5 & 1.3 & 14.0 & 3.5 & 2.3 & 3.5 & 3.5 \\
\hline 29 & Tilia tomentosa Mnch. & 2.5 & 1.1 & 6.6 & 1.4 & 1.4 & 3.9 & 4.0 \\
\hline
\end{tabular}

The average vitality score of woody species was 3.9. As many as 234 trees received the highest vitality rating (4 and 5). The species with the mean value of the vitality rating above the average were: Abies alba, Acer campestre, Acer pseudoplatanus, Betula verrucosa, Cedrus atlantica, Cupressus sempervirens, Fraxinus angustifolia, Koelreuteria paniculata, Picea omorika, Picea pungens, Pinus nigra, Platanus acerifolia, Prunus avium, Prunus pissardii, Quercus rubra, and Thuja orientalis.

Of the registered deciduous species, Cercis siliquastrum got the lowest average vitality rating, while Juniperus scopulorum 'Skyrocket' and Libocedrus decurrens were the conifers with the lowest vitality rating. 
The ornamental value of woody species was scored with the average rating of 3.8. As many as 49 trees received the maximum rating for their ornamental value. The following species were rated 5: Prunus avium (17 trees), Chamaecyparis lawsoniana (10), Libocedrus decurrens (4), Juniperus scopulorum 'Skyrocket', Quercus rubra and Tilia tomentosa (3 trees each), Betula verrucosa, Prunus pissardii and Thuja orientalis (2 trees each) and Cedrus atlantica, Pinus nigra and Platanus acerifolia (1 tree each).

The species with both the vitality score and the ornamental value score above the average were: Abies alba, Acer campestre, Cupressus sempervirens, Fraxinus angustifolia, Picea omorika, Pinus nigra, Platanus acerifolia, Prunus avium, Prunus pissardii, Quercus rubra and Thuja orientalis.

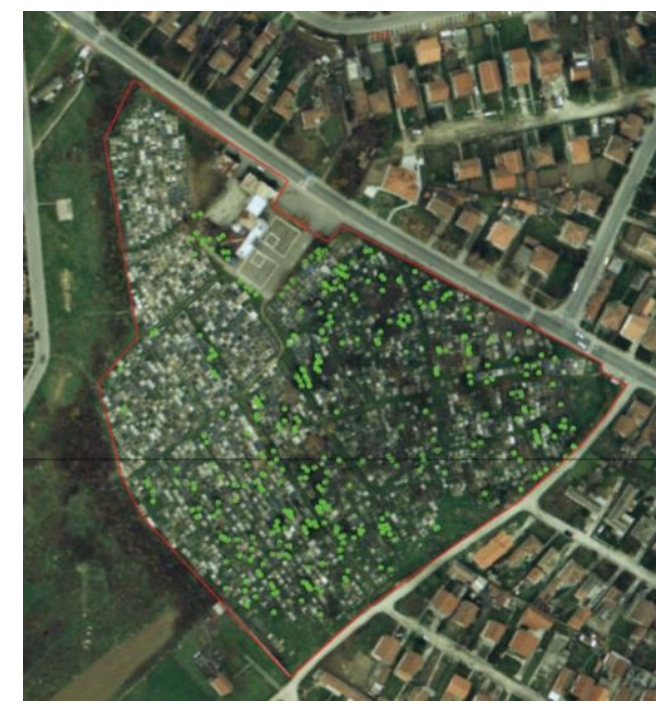

Figure 3. The position of the Old Cemetery

The Old Cemetery had 348 trees analyzed (Figure 3), 53 of which were broadleaves and 295 conifers. There were 32 different tree species in total. The most common genus was Picea sp. with 3 species ( $P$. abies, P. omorika, P. pungens). By far the most abundant woody species was Thuja orientalis with 166 trees. The ratio of broadleaves to conifers was15:85.

Table 2. Average values of the recorded tree parameters in the Old Cemetery

\begin{tabular}{|c|c|c|c|c|c|c|c|c|}
\hline $\begin{array}{l}\text { S. } \\
\mathrm{n}^{\circ}\end{array}$ & Species & 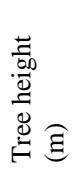 & 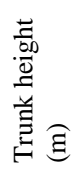 & 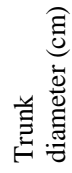 & 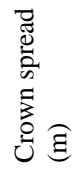 & 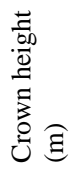 & 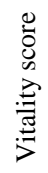 & 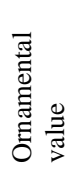 \\
\hline 1 & Abies alba Mill. & 15.0 & 2.5 & 23.0 & 5.0 & 12.5 & 3.0 & 4.0 \\
\hline 2 & Betula verrucosa Ehrh. & 10.5 & 2.9 & 24.3 & 5.6 & 7.7 & 3.7 & 3.7 \\
\hline 3 & Carpinus betulus L. & 16.5 & 2.1 & 32.5 & 6.8 & 14.4 & 3.7 & 3.7 \\
\hline 4 & Catalpa bignonioides Walt. & 9.0 & 2.1 & 25.5 & 7.8 & 6.9 & 4.5 & 4.5 \\
\hline 5 & Cedrus atlantica Man. & 18.5 & 5.2 & 38.0 & 9.0 & 13.3 & 4.0 & 4.0 \\
\hline 6 & Chamaecyparis lawsoniana (Murr.) Parl & 8.6 & 2.4 & 19.4 & 3.6 & 6.3 & 3.3 & 3.5 \\
\hline 7 & Cupressus arizonica Greene & 10.0 & 1.9 & 24.7 & 4.2 & 8.1 & 3.3 & 3.4 \\
\hline
\end{tabular}




\begin{tabular}{|c|c|c|c|c|c|c|c|c|}
\hline $\begin{array}{l}\text { S. } \\
n^{\circ}\end{array}$ & Species & 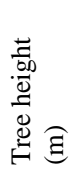 & 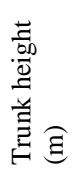 & 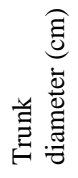 & 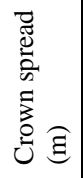 & 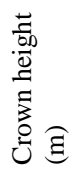 & 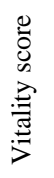 & 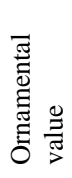 \\
\hline 8 & Cupressus sempervirens $\mathrm{L}$. & 10.3 & 2.0 & 21.9 & 3.4 & 8.3 & 3.3 & 3.4 \\
\hline 9 & Gleditsia triacanthos $\mathrm{L}$. & 13.6 & 2.5 & 29.4 & 7.0 & 11.1 & 4.0 & 4.0 \\
\hline 10 & Juglans regia $\mathrm{L}$. & 11.8 & 3.3 & 42.8 & 8.8 & 8.6 & 3.3 & 3.3 \\
\hline 11 & Juniperus excelsa Bieb. & 5.2 & 1.4 & 16.0 & 3.3 & 3.8 & 3.3 & 3.3 \\
\hline 12 & Juniperus scopulorum 'Skyrocket' Sarg. & 4.7 & 0.7 & 9.5 & 1.3 & 4.0 & 2.8 & 3.1 \\
\hline 13 & Libocedrus decurrens Torr. & 5.0 & 2.2 & 8.0 & 2.8 & 2.8 & 3.0 & 4.0 \\
\hline 14 & Magnolia $x$ soulangeana Soul.-Bod. & 5.7 & 1.5 & 14.3 & 5.0 & 4.2 & 3.3 & 3.3 \\
\hline 15 & Malus sp. Mill. & 5.0 & 2.0 & 11.0 & 3.5 & 3.0 & 3.5 & 3.0 \\
\hline 16 & Picea abies (L.) Karst. & 6.8 & 2.2 & 16.1 & 4.0 & 4.6 & 2.8 & 2.7 \\
\hline 17 & Picea omorika (Pančić) Purkyne. & 6.8 & 3.4 & 12.5 & 2.0 & 3.4 & 4.0 & 4.0 \\
\hline 18 & Picea pungens Engelm. & 7.0 & 2.8 & 17.7 & 3.7 & 4.2 & 3.3 & 3.3 \\
\hline 19 & Pinus nigra Arn. & 9.2 & 2.8 & 25.7 & 5.5 & 6.4 & 3.7 & 3.7 \\
\hline 20 & Pinus sylvestris $\mathrm{L}$. & 13.3 & 4.7 & 41.0 & 6.5 & 8.7 & 3.3 & 3.7 \\
\hline 21 & Prunus avium L. & 8.0 & 1.3 & 25.0 & 4.8 & 6.8 & 3.5 & 3.5 \\
\hline 22 & Prunus cerasus Ehrh. & 5.0 & 1.7 & 14.0 & 3.7 & 3.3 & 3.3 & 3.3 \\
\hline 23 & Prunus persica (L.) Batsch & 3.0 & 1.7 & 6.0 & 3.0 & 1.3 & 3.0 & 3.0 \\
\hline 24 & Pseudotsuga menziesii (Mirbel.) Franco & 9.8 & 2.6 & 21.8 & 4.3 & 7.2 & 3.2 & 3.5 \\
\hline 25 & Pyrus communis $\mathrm{L}$. & 5.3 & 1.7 & 17.3 & 4.0 & 3.7 & 3.3 & 3.3 \\
\hline 26 & Quercus robur $\mathrm{L}$. & 17.0 & 2.5 & 36.5 & 7.0 & 14.5 & 3.0 & 3.0 \\
\hline 27 & Syringa vulgaris $\mathrm{L}$. & 3.0 & 0 & 6.0 & 2.0 & 3.0 & 3.0 & 3.0 \\
\hline 28 & Taxus baccata $\mathrm{L}$. & 6.0 & 0.5 & 24.0 & 5.0 & 5.5 & 4.0 & 4.0 \\
\hline 29 & Thuja occidentalis L. & 7.9 & 2.1 & 22.8 & 3.7 & 5.8 & 3.3 & 3.8 \\
\hline 30 & Thuja orientalis $\mathrm{L}$. & 5.9 & 1.6 & 13.9 & 3.3 & 4.2 & 3.1 & 3.2 \\
\hline 31 & Tilia cordata Mill. & 9.9 & 2.8 & 30.6 & 8,0 & 7.1 & 2.8 & 3.6 \\
\hline 32 & Tilia tomentosa Mnch. & 17.0 & 2.6 & 75.7 & 11.7 & 14.4 & 4.0 & 4.0 \\
\hline
\end{tabular}

The average score of the vitality of woody species was 3.4. The following species received the maximum average vitality rating (4): Cedrus atlantica, Gleditsia triacanthos, Picea omorika and Tilia tomentosa (species with one or two individuals on the green area were excluded from the study). Two trees had their vitality rated 5 - Catalpa bignonioides and Chamaecyparis lawsoniana.

The species that had the mean score of vitality above the average were: Betula verrucosa, Carpinus betulus, Catalpa bignonioides, Cedrus atlantica, Gleditsia triacanthos, Malus sp., Picea omorika, Pinus nigra, Prunus avium, Taxus baccata and Tilia tomentosa.

The ornamental value of woody species was scored with the average rating of 3.5. The above-mentioned species with the maximum average vitality rating (4) also had the maximum average ornamental value rating (4). In total, 5 trees received the rating 5. Besides the common catalpa and Lawson cypress, 3 trees of Thuja orientalis had the greatest ornamental value. The following species had the mean rating of the ornamental value above the average: Abies alba, Betula verrucosa, Carpinus betulus, Catalpa bignonioides, Cedrus atlantica, Gleditsia triacanthos, Libocedrus decurrens, Picea omorika, Pinus nigra, Pinus sylvestris, Taxus baccata, Thuja occidentalis, Tilia cordata and Tilia tomentosa.

The species with both the vitality score and the ornamental value score above the average were: Betula verrucosa, Carpinus betulus, Catalpa bignonioides, Cedrus atlantica, Gleditsia triacanthos, Picea omorika, Pinus nigra, Taxus baccata and Tilia tomentosa. 
Table 3. The average values of the registered parameters of tree species present at both the New Cemetery and the Old Cemetery

\begin{tabular}{|c|c|c|c|c|c|c|c|c|c|}
\hline Loc. & Species & 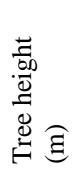 & 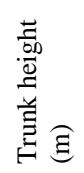 & 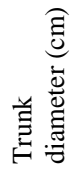 & 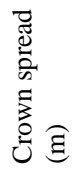 & 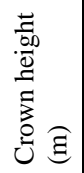 & 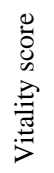 & 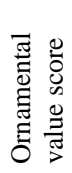 & 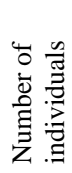 \\
\hline New & \multirow{2}{*}{ Abies alba } & 1.5 & 0.0 & 3.0 & 1.0 & 1.5 & 4.0 & 4.0 & 2 \\
\hline Old & & 15.0 & 2.5 & 23.0 & 5.0 & 12.5 & 3.0 & 4.0 & 1 \\
\hline New & \multirow{2}{*}{ Betula verrucosa } & 3.0 & 0.5 & 6.7 & 2.0 & 2.4 & 4.0 & 3.8 & 37 \\
\hline Old & & 10.5 & 2.9 & 24.3 & 5.6 & 7.7 & 3.7 & 3.7 & 6 \\
\hline New & \multirow{2}{*}{ Catalpa bignonioides } & 2.0 & 1.5 & 4.0 & 1.0 & 0.5 & 3.7 & 3.9 & 10 \\
\hline Old & & 9.0 & 2.1 & 25.5 & 7.8 & 6.9 & 4.5 & 4.5 & 2 \\
\hline New & \multirow{2}{*}{ Cedrus atlantica } & 2.3 & 0.0 & 7.3 & 1.4 & 2.3 & 4.3 & 3.7 & 3 \\
\hline Old & & 18.5 & 5.2 & 38.0 & 9.0 & $\begin{array}{ll}13.3 \\
\end{array}$ & 4.0 & 4.0 & 4 \\
\hline New & \multirow{2}{*}{ Chamaecyparis lawsoniana } & 2.1 & 0.0 & 5.4 & 1.1 & 2.1 & 3.7 & 3.6 & 32 \\
\hline Old & & 8.6 & 2.4 & 19.4 & 3.6 & 6.3 & 3.3 & 3.5 & 22 \\
\hline New & \multirow{2}{*}{ Cupressus sempervirens } & 5.0 & 0.0 & 10.0 & 1.0 & 5.0 & 4.0 & 4.0 & 1 \\
\hline Old & & 10.3 & 2.0 & 21.9 & 3.4 & 8.3 & 3.3 & 3.4 & 12 \\
\hline New & \multirow{2}{*}{ Juglans regia } & 7.0 & 1.5 & 41.0 & 7.0 & 5.5 & 3.0 & 3.0 & 1 \\
\hline Old & & 11.8 & 3.3 & 42.8 & 8.8 & 8.6 & 3.3 & 3.3 & 6 \\
\hline New & \multirow{2}{*}{ Juniperus scop. 'Skyrocket' } & 2.7 & 0.1 & 5.4 & 0.8 & 2.6 & 3.4 & 3.9 & 7 \\
\hline Old & & 4.7 & 0.7 & 9.5 & 1.3 & 4.0 & 2.8 & 3.1 & 11 \\
\hline New & \multirow{2}{*}{ Libocedrus decurrens } & 2.6 & 0.0 & 8.1 & 1.2 & 2.6 & 3.4 & 4.3 & 11 \\
\hline Old & & 5.0 & 2.2 & 8.0 & 2.8 & 2.8 & 3.0 & 4.0 & 2 \\
\hline New & \multirow{2}{*}{ Picea abies } & 3.8 & 0.3 & 12.5 & 2.3 & 3.5 & 3.5 & 3.5 & 2 \\
\hline Old & & 6.8 & 2.2 & 16.1 & 4.0 & 4.6 & 2.8 & 2.7 & 29 \\
\hline New & \multirow{2}{*}{ Picea omorika } & 2.0 & 0.0 & 7.0 & 1.0 & 2.0 & 4.0 & 4.0 & 1 \\
\hline Old & & 6.8 & 3.4 & 12.5 & 2.0 & 3.4 & 4.0 & 4.0 & 4 \\
\hline New & \multirow{2}{*}{ Picea pungens } & 2.5 & 0.0 & 5.0 & 1.2 & 2.5 & 4.0 & 3.0 & 1 \\
\hline Old & & 7.0 & 2.8 & 17.7 & 3.7 & 4.2 & 3.3 & 3.3 & 3 \\
\hline New & \multirow{2}{*}{ Prunus avium } & 1.9 & 0.7 & 4.0 & 1.4 & 1.2 & 4.8 & 4.7 & 20 \\
\hline Old & & 8.0 & 1.3 & 25.0 & 4.8 & 6.8 & 3.5 & 3.5 & 2 \\
\hline New & \multirow{2}{*}{ Thuja orientalis } & 1.8 & 0.0 & 4.3 & 0.7 & 1.8 & 4.1 & 4.1 & 13 \\
\hline Old & & 5.9 & 1.6 & 13.9 & 3.3 & 4.2 & 3.1 & 3.2 & 166 \\
\hline New & \multirow{2}{*}{ Tilia cordata } & 3.5 & 1.3 & 14.0 & 3.5 & 2.3 & 3.5 & 3.5 & 2 \\
\hline Old & & 9.9 & 2.8 & 30.6 & 8.0 & 7.1 & 2.8 & 3.6 & 5 \\
\hline New & \multirow{2}{*}{ Tilia tomentosa } & 2.5 & 1.1 & 6.6 & 1.4 & 1.4 & 3.9 & 4.0 & 40 \\
\hline Old & & 17.0 & 2.6 & 75.7 & 11.7 & 14.4 & 4.0 & 4.0 & 6 \\
\hline
\end{tabular}

Out of the total of 45 recorded species, 16 species occurred both in the New Cemetery and in the Old Cemetery. Only two coniferous species, Picea omorika and Pinus nigra, had the scores of vitality and ornamental value above the average at both sites. The average values of the mean tree and trunk heights, the diameter and crown spread were higher in the species at the site of the Old Cemetery, which was expected because the trees were older. On the other hand, the trees in the New Cemetery site had higher average scores of vitality and ornamental value because they were young and properly tended.

\section{CONCLUSIONS}

There are 45 species of trees identified at Obrenovac cemeteries. 15 of them are coniferous and 30 are broadleaved. The New Cemetery has 11 coniferous and 28 broadleaved species, while the Old Cemetery has 17 coniferous and 15 
broadleaved species. Some specimens in the Old Cemetery have exceptional aesthetic value and represent true natural monuments. The diversity of species adds to the richness of colours of the area throughout the whole year.

The percentage ratio of broadleaved and coniferous species in the New Cemetery is 64:36, while it is 15:85 in the Old Cemetery.

Woody species that have the highest ratings are Picea omorika and Pinus nigra, so it can be concluded that they are best adapted to the environmental conditions in both cemeteries. In the New Cemetery, the species Abies alba, Acer campestre, Cupressus sempervirens, Fraxinus angustifolia, Platanus acerifolia, Prunus avium, Prunus pissardii, Quercus rubra and Thuja orientalis have shown extremely great adaptability. Based on the assessment of vitality and ornamental value, it can be concluded that Betula verrucosa, Carpinus betulus, Catalpa bignonioides, Cedrus atlantica, Gleditsia triacanthos, Taxus baccata and Tilia tomentosa have shown the best adaptability in the Old Cemetery.

Based on the above, it can be concluded that there are a large number of coniferous and broadleaved trees at the sites of the New and Old Cemeteries in Obrenovac, most of which have exceptional ornamental properties and represent special features of the green area of the urban core of Obrenovac.

\section{REFERENCES}

Anastasijević, N. (2002): Establishment and cultivation of green areas, Faculty of Forestry, University of Belgrade, Belgrade, 248 (In original: Anastasijević, N. (2002): Podizanje $i$ negovanje zelenih površina. Šumarski fakultet Univerziteta u Beogradu, Beograd, 248)

Ćirković-Mitrović T., Brašanac-Bosanac Lj., Mladenović K., Milenković I (2016): The Comparative analysis of the state of dendrological plants in Belgrade parks, Serbia. Sustainable Forestry, Collection 73-74. Institute of Forestry, Belgrade, 11-18.

Hessayon, D.G. (2001): The Flowering Shrub Expert, Mozaik Knjiga, Zagreb, 128 (In original: Hessayon, D.G. (2001): Cvatući grmovi, Mozaik knjiga, Zagreb, 128).

Hillier, H.G. (1982): Color dictionary of Trees and Shrubs. Van Nostrand Reinhold Company, New York, 323.

Idjojtić, M. (2009): Dendrology Journal. Faculty of Forestry, University of Zagreb, Zagreb, 903. (In original: Idžojtić, M. (2009): Dendrologija list, Šumarski fakultet Sveučilišta u Zagrebu, Zagreb, 903).

Institute of Soil Science (2009): Study on the degree of vulnerability of soil and water to hazardous and harmful substances in local communities on the periphery of ash dumps in the CM Obrenovac, Belgrade. (In original: Institut za zemljište (2009): Studija o stepenu ugroženosti zemljišta $i$ voda opasnim $i$ štetnim materijama u MZ na obodu deponija pepela na području GO Obrenovac, Beograd).

Jovanović, B. (1985): Dendrology, Faculty of Forestry, University of Belgrade, Belgrade, 557 (In original: Jovanović, B. (1985): Dendrologija. Šumarski fakultet Univerziteta u Beogradu, Beograd, 557). 
Ocokoljić, M., Ninić-Todorović, J. (2003): A Handbook of Ornamental Dendrology, Faculty of Forestry, University of Belgrade, Belgrade, 162. (In original: Ocokoljić, M., Ninić-todorović, J. (2003): Priručnik iz dekorativne dendrologije. Šumarski fakultet Univerziteta u Beogradu, Beograd, 162).

Dragičević, S., Karić I. (2007): Geospatial characteristics of the urban municipality of Obrenovac. Environmental Protection Fund Obrenovac, Obrenovac. (In original: Dragičević, S., Karić I. (2007): Geoprostorne karakteristike gradske opštine obrenovac. Fond za zaštituu životne sredine Obrenovac, Obrenovac)

Veselinović M., Mitrović S., Radulović Z., Dražić D., Čule N., Stanković D., Rajković S. (2014) The condition of Aesculus hippocastanum L. trees in the avenues of the central part of the city of Obrenovac. Sustainable Forestry, Collection 69-70. Institute of Forestry, Belgrade, 17-23.

Veselinović, M., Čule, N., Mitrović, S. (2010): The cadastre of public green areas in the urban area of the Municipality of Obrenovac integrated into the GIS, Institute of Forestry, Belgrade, 107 (In original: Veselinović, M., Čule, N., Mitrović, S. (2010): Katastar zelenih površina javnog karaktera gradskog područja opštine Obrenovac integrisanih u GIS. Institut za šumarstvo, Beograd, 107)

Vidaković, M. (1982): Conifers: morphology and variability. JAZU, University Press Liber, Zagreb, 707 (In original: Vidaković, M. (1982): Četinjače: morfologija $i$ varijabilnost. JAZU, Sveučilišna naklada Liber, Zagreb, 707)

Vujković, Lj. (2003): Landscape Architecture Planning and Design, Faculty of Forestry, University of Belgrade, Belgrade, 224 (In original: Vujković, Lj. (2003): Pejzažna arhitektura planiranje $i$ projektovanje. Šumarski fakultet Univerziteta u Beogradu, Beograd, 224).

Vukićević, E. (1996): Ornamental, Faculty of Forestry, University of Belgrade, Belgrade, 585 (In original: Vukićević, E. (1996): Dekorativna dendrologija, Šumarski fakultet Univerziteta u Beogradu, Beograd, 585).

Šijak, M. (2000): Multilingual Dendrological Dictionary: Vocabularium dendrologicum multilinguum. Faculty of Forestry, 310 (In original: Šijak, M. (2000): Višejezični dendrološki rečnik: Vocabularium dendrologicum multilinguum. Šumarski fakultet, 310)

\title{
THE STATE AND FUNCTIONALITY OF DENDROFLORA IN THE CEMETERIES IN OBRENOVAC
}

\author{
Suzana MITROVIĆ, Nevena ČULE, Dušan JOKANOVIĆ, Milijana CVEJIĆ, \\ Milorad VESELINOVIĆ
}

\section{Summary}

Cemeteries are important elements of urban areas, with multiple benefits in terms of environmental protection, connected with other green areas of different categories, constitute the green structure of the city whose functions contribute to the healthier environment of the polluted urban area. 
There are two urban cemeteries on the territory of the urban area of the Municipality of Obrenovac. The New Cemetery is located in the western part of Obrenovac, $2.84 \mathrm{~km}$ away from the city center, covers an area of 5.4 ha. The Old Cemetery, measuring 4.5 hectares, located in the western part of Obrenovac, $2 \mathrm{~km}$ from the city center.

In the New Cemetery, 271 trees were analyzed, 174 of which were broadleaved and 97 coniferous species. There were 29 different tree species in total. The most common wood species were Tilia tomentosa Mnch. with 40 individuals, Betula verrucosa Ehrh. with 37 seedlings and Chamaecyparis lawsoniana (Murr.) Parl. with 28 specimens. The ratio of the broadleaves to conifers was 64:36.

The Old Cemetery had 348 trees analyzed, 53 of which were broadleaves and 295 conifers. There were 32 different tree species in total. The most common genus was Picea sp. with 3 species ( $P$. abies, $P$. omorika, P. pungens). By far the most abundant woody species was Thuja orientalis with 166 trees. The ratio of broadleaves to conifers was 15:85.

Woody species that have the highest ratings are Picea omorika and Pinus nigra, so it can be concluded that they are best adapted to the environmental conditions in both cemeteries. In the New Cemetery, the species Abies alba, Acer campestre, Cupressus sempervirens, Fraxinus angustifolia, Platanus acerifolia, Prunus avium, Prunus pissardii, Quercus rubra and Thuja orientalis have shown extremely great adaptability. Based on the assessment of vitality and ornamental value, it can be concluded that Betula verrucosa, Carpinus betulus, Catalpa bignonioides, Cedrus atlantica, Gleditsia triacanthos, Taxus baccata and Tilia tomentosa have shown the best adaptability in the Old Cemetery.

In the area of research there are a large number of coniferous and broadleaved trees at the sites, most of which have exceptional ornamental properties and represent special features of the green area of the urban core of Obrenovac.

\title{
STANJE I FUNKCIONALNOST DENDROFLORE NA GROBLJIMA U OBRENOVCU
}

\author{
Suzana MITROVIĆ, Nevena ČULE, Dušan JOKANOVIĆ, Milijana CVEJIĆ, \\ Milorad VESELINOVIĆ
}

\section{Rezime}

U gradskim sredinama, groblja predstavljaju značajne objekte sa svojim opštekorisnim funkcijama u smislu zaštite životne sredine povezane sa ostalim zelenim površinama različitih kategorija, i sačinjavaju zelenu strukturu grada koja svojim funkcijama utiče na zdravije okruženje zagađene gradske sredine.

$\mathrm{Na}$ teritoriji gradskog područja opštine Obrenovac nalaze se dva gradska groblja. Novo groblje se nalazi u zapadnom delu Obrenovca, 2,84 km od centra grada, ukupne površine 5,4 ha. Staro groblje, površine 4,5 hektara, se nalazi u zapadnom delu Obrenovca, $2 \mathrm{~km}$ od centra grada.

Terenskim istraživanjima na području gradskih grobalja drveće je evidentirano: na Novom groblju je analizirano 271 stabala, od čega je 174 lišćarskih i 97 četinarskih vrste. Ukupno postoji 29 različitih vrsta drveća. Najzastupljenije drvenaste vrste su Tilia tomentosa Mnch. sa 40 jedinki, Betula verrucosa Ehrh. sa 37 sadnica i Chamaecyparis lawsoniana (Murr.) Parl. sa 28 primeraka. Odnos lišćara i četinara je 64:36.

Na Starom groblju je analizirano 348 stabala, od toga 53 lišćara i 295 četinara. Ukupno postoji 32 različitih vrsta drveća. Najprisutniji je rod Picea sp. sa 3 vrste (P. abies, $P$. omorika, $P$. pungens). Ubedljivo najzastupljenija drvenasta vrsta je Thuja orientalis sa 166 stabala. Odnos lišćara i četinara je 15:85. 
Drvenaste vrste koje imaju najviše ocene su Picea omorika i Pinus nigra, i može se konstatovati da su one najbolje adaptirane na uslove sredine na oba groblja. Na Novom groblju vrste: Abies alba, Acer campestre, Cupressus sempervirens, Fraxinus angustifolia, Platanus acerifolia, Prunus avium, Prunus pissardii, Quercus rubra i Thuja orientalis su pokazale izuzetno veliku adaptivnost. $\mathrm{Na}$ osnovu ocena vitalnosti i dekorativnosti može zaključiti da su vrste: Betula verrucosa, Carpinus betulus, Catalpa bignonioides, Cedrus atlantica, Gleditsia triacanthos, Taxus baccata i Tilia tomentosa, pokazale najbolju adaptivnost na Starom groblju.

$\mathrm{Na}$ analiziranim grobljima zastupljen je veliki broj četinarskih i lišćarskih vrsta drveća, od kojih većina imaju izuzetna dekorativa svojstva i predstavljaju značaje zelene površine gradskog jezgra Obrenovca. 